\title{
Oncology 2015: new therapies and new transitions toward value-based cancer care
}

\author{
Linda D Bosserman, MD, FACP
}

T The past year has been an exciting one for new oncology and hematology drug approvals and the continued evolution of our oncology delivery system toward high quality and value. In all, at press time in mid-November, the US Food and Drug Administration (FDA) had approved or granted expanded indications for 24 drugs, compared with 19 in the 2 preceding years. Of those 24 approvals, 7 were accelerated and 6 were expanded approvals, and 3 alone were for the immunotherapeutic drug, nivolumab 2 for non-small-cell lung cancer (NSCLC) and 1 for metastatic melanoma.

Most notable among the approvals were more immunotherapy drugs targeting the PD-1 and PD-L1 pathways for different tumor types and approvals for combinations of those antibodies, but with further sticker shock on exorbitant costs for both

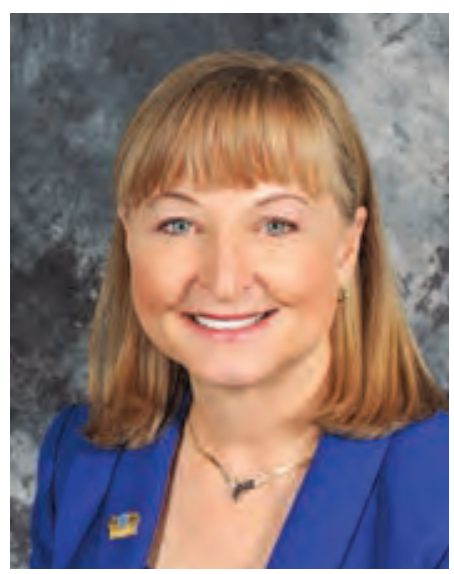

Act, which amended the Public Health Service Act. This created a licensure pathway for biologic products that can be shown to be biosimilar to or interchangeable with an FDA-licensed biologic product. Analysts are predicting that biosimilars will initially help reduce costs by $15 \%$ $20 \%$ over the originator drug. Over time, as manufacturing capacities and techniques are refined, it is hoped that a growing array of biologic drugs can be produced and provided at even lower costs.

\section{New options, but some costs are prohibitive}

Drugs were approved or had expanded indications for the very rare metastatic basal cell carcinoma; liposarcomas and metastatic, well-differentiated thyroid cancers; and Waldenstrom's macroglobulinemia, as well as the more common cancers of breast, lung, payers and patients. These biologic drugs are routinely running $\$ 100,000-\$ 150,000$ for a course of therapy, and combinations run even higher. The rising costs, variable benefits, and growing call for value assessments were topics of discussion at medical meetings during the year, and were the subject of a major plenary session at this year's annual meeting of the American Society of Clinical Oncology (ASCO). Even the oral drugs have become shockingly expensive. For example, the new oral CK4/6 inhibitor pathway drug, palbociclib, for a common subtype of breast cancer is an exciting addition to an effective oral regimen, but it runs to $\$ 10,000-\$ 12,000$ a month. For patients with limited oral chemotherapy coverage, even the generous pharmaceutical company copay assistance programs have meant several of my patients had to choose more toxic intravenous therapies because they could not afford the monthly copay for preferred oral regimens.

On the brighter side, however, was the approval of filgrastim-sndz (Zarxio) for the same indications as its originator or reference drug, filgrastaim (Neupogen; see pp 420 and 418) - after more than 7.5 million doses had been given in Europe. It was the first biosimilar drug approved in the United States under the section of the Patient Protection and Affordable Care Act of March 23, 2010 called the Biologics Price Competition and Innovation colorectal, melanoma, myeloma, and pancreas. Although it is exciting to have more effective therapy choices, the continued rise in cost as each new drug or class of drug comes to market is straining patients' ability to pay and payers' ability to control the level of premium increases, which are once again threatening people's ability to maintain health insurance coverage. Among the approvals of new oncology drugs were:

- Trabectedin (Yondelis) for patients with unresectable or metastatic liposarcoma or leiomyosarcoma who have received a prior anthracycline-containing regimen;

- Liposomal irinotecan (Onivyde) in combination with fluorouracil (5FU) and leucovorin, for patients with metastatic adenocarcinoma of the pancreas whose disease has progressed following gemcitabine-based therapy;

- Nivolumab (Opdivo) for patients with metastatic squamous NSCLC with progression on or after platinumbased chemotherapy. It was also approved for 2 expanded indications: in combination with ipilimumab for patients with BRAF V600 wild-type, unresectable or metastatic melanoma; and for those with metastatic NSCLC with progression on or after platinum-based chemotherapy;

- Trifluridine/tipiracil (Lonsurf) for patients with metastatic colorectal cancer $(\mathrm{mCRC})$ previously treated with the anti-VEGF [vascular endothelial growth fac- 
tor] biologic fluoropyrimidine-, oxaliplatin-, and irinotecan-based chemotherapy and an anti-EGFR [epidermal growth factor receptor] monoclonal antibody, if RAS wild-type;

- Sonidegib (Odomzo) for patients with locally advanced basal-cell carcinoma recurrence after surgery or radiation therapy, or those who are not candidates for surgery or radiation therapy;

- Ramucirumab (Cyramza) for use with the FOLFIRI regimen (folinic acid, 5FU, irinotecan) for the patients with $\mathrm{mCRC}$ whose disease has progressed on a firstline regimen containing bevacizumab, oxaliplatin, and fluoropyrimidine;

- Lenvatinib (Lenvima) for locally recurrent or metastatic, progressive, radioactive iodine-refractory differentiated thyroid cancer;

- Gefitinib (Iressa) for patients with metastatic NSCLC whose tumors have EGFR exon 19 deletions or exon 21 (L858R) substitution mutations as detected by the therascreen EGFR RG QPCR companion diagnostic test for patient selection, also approved by the FDA;

- Palbociclib (Ibrance) for use in combination with letrozole for treating postmenopausal women with estrogen receptor-positive, human epidermal growth factor receptor-2-negative advanced breast cancer as initial endocrine-based therapy for their metastatic disease;

- Ibrutinib (Imbruvica) received an expanded indication for the treatment of patients with Waldenstrom's macroglobulinemia;

- Panobinostat (Fardak) received an accelerated approval in combination with bortezomib and dexamethasone for patients with multiple myeloma who have received at least 2 prior regimens, including bortezomib and an immunomodulatory agent;

- Cobimetinib (Cotellic) for patients with unresectable or metastatic melanoma with BRAF V600E or V600K mutation, in combination with vemurafenib; and

- Osimertinib (Tagrisso; previously known as AZD9291) for patients with metastatic EGFR T790M mutationpositive NSCLC, as detected by an FDA-approved test, who have progressed on or after EGFR tyrosine kinase inhibitor therapy.

New hematology drugs also added important therapeutic options for pediatric patients and for Hodgkin lymphoma patients after bone marrow transplant. Of particular note was the approval of idarucizumab, the first drug to reverse the anticoagulant effects of dabigatran, a popular, safe, and effective drug that had raised concerns if emergency or urgent reversal was needed. The new hematology drugs approved were:

- Idarucizumab (Praxbind) for patients treated with dabigatran when the anticoagulant effects of dabigatran need to be reversed for emergency surgery or urgent proce- dures, or in life-threatening or uncontrolled bleeding;

- Eltrombopag (Promacta) for the treatment of thrombocytopenia in pediatric patients aged 1 year or older with chronic immune idiopathic thrombocytopenia with insufficient response to corticosteroids, immunoglobulins, or splenectomy;

- Brentuximab vedotin (Adcetris) for the postautologous hematopoietic stem-cell transplantation consolidation treatment of patients with classical Hodgkin lymphoma who are at high risk of relapse or progression; and

- Dinutuximab (Unituxin) in combination with granulocyte-macrophage colony-stimulating factor, interleukin-2, and 13-cis-retinoic acid, for pediatric patients with high-risk neuroblastoma who achieve at least a partial response to prior first-line multiagent, multimodality therapy.

Three other drugs with expanded indications were:

- Ipilimumab (Yervoy) as an adjuvant treatment for patients with cutaneous melanoma with pathologic involvement of regional lymph nodes of more than $1 \mathrm{~mm}$ who have undergone complete resection;

- Pembrolizumab (Keytruda) for patients with metastatic NSCLC whose tumors express PD-L1 (based on an FDA-approved test) and who have had disease progression on or after platinum-containing chemotherapy; and

- Carfilzomib (Kyprolis) with lenalidomide and dexamethasone for patients with relapsed multiple myeloma who have received 1-3 prior lines of therapy.

\section{Toward value-based cancer care}

On the practice front, 2015 saw the continued shift away from volume-based services, delivery strategies, and payments toward services that can deliver and document highvalue, high-quality oncology care. A number of recent developments have made the move to reportable, evaluable, value-based cancer care a national priority, including cutting-edge diagnostics and tumor subtyping and the resultant use of more targeted therapies; our access to complex, real-time analytics of predicted benefits for individual patients; and the skyrocketing costs of drug. Patients, payers, and employers want to know that the best possible care is being delivered for meaningful patient, clinical, and financial outcomes.

As the United States is asked to spend a greater share of the GDP on health care, people are eager to ensure they understand the benefits of that investment against other valuable national investments in education, infrastructure, and research. Pathways as tools for guiding physicians in best practices are starting to be used more widely as busy clinicians recognize the need for easily accessible, sciencebased guidelines to help them in their decisions about increasingly complex molecular tests, specific therapies, and sequencing of therapies as they strive to achieve optimum 
health outcomes. In June, the ASCO Value Framework for assessing the value of new cancer therapies based on their clinical benefits, toxicities, and costs was released for public comment. The framework, which will eventually incorporate stakeholder feedback, will allow clinicians to use data on the benefits and toxicities of a regimen to calculate a health benefit score, or NHB, for a new drug so that they can compare it with an existing standard-of-care therapy. ASCO also launched CancerLinQ, a health-information system based on the collection and analysis of real-world patient data. The system can help practices gauge how their care measures up against that of other practices, and physicians in selecting therapies for patients and learning about previously unknown patterns of treatments, patient characteristics, and outcomes, all with the goal of improving care. In addition, the Centers for Medicare and Medicaid Services chose applicants for its Oncology Care Model $(\mathrm{OCM})$ pilot, and large regional accountable care organization groups continued to share results from early oncology medical home pilots with new payment models.
This year, more private practices across the country joined larger community or community academic networks to work more effectively with large payers on value-based contracting and other value-based pilots such as the OCM. We can truly envision a time in the next few years when a practice or network has precision medicine information and value options at the point-of-care delivery to support patient-shared decision making with integrated data collection and real-time clinical and financial analytics on what works best for which patients, which comorbidities, and personal life goals.

On behalf of my co-editors and the professional staff of our journal, we remain grateful for your contributions, feedback and support as we continue our mission to provide clinically relevant, scientifically rigorous, up-to-date oncology articles with a practical focus. From our oncology family to yours, we wish you the best of the holiday season and a new year of joyful fulfillment in your professional and personal lives. 